\title{
B04 Seismic Monitoring of Land Reservoirs and Correlation with Control Well Pressure Data
}

\author{
J. Bruneau, IFP, F. Huguet, GDF, E. Bathellier, E. Forgues, J. Meunier and S. Rodriguez, \\ CGGVeritas
}

In partnership with Gaz de France (GDF) and Institut Français du Pétrole (IFP), CGGVeritas developed SeisMovie ${ }^{\mathrm{TM}}$, an extremely flexible, non-stop monitoring system based on a network of stationary low-energy piezoelectric seismic sources and multi-component receiver arrays, either vertical or horizontal. The system, which is buried below the weathering layer, can work continuously or on demand and is aimed at being fully autonomous and remotely controlled. The low instantaneous emitted energy is compensated by long emission times and by low ambient noise in the receiving area. Highly reliable and repeatable, we believe that the SeisMovie ${ }^{\mathrm{TM}}$ system can provide seismic based reservoir information which is complementary to pressure curves obtained from control wells. To optimize emission time in case of multi-sources applications, all sources emit simultaneously, providing a very high temporal information density.

Early results, obtained over a multi-year period from the underground gas storage at Germignysous-Coulombs (Deflandre et al., 1995) and Céré-la-Ronde (Meunier et Huguet, 1998) were promising. In 2002, a 4 years SeisMovie ${ }^{\mathrm{TM}}$ prototype experience at the Saint-Clair-sur-Epte gas storage (Bianchi et al., 2004) has started. The system comprised five permanent piezoelectric sources located above multi-component receiver vertical arrays including hydrophones and geophones. A PC-based electronic system controls the sources, triggered the recording, records and pre-processes the seismic data and transmits them to a central station. This key demonstration showed how sensitive and precise seismic time-lapse monitoring can be, on land, if we keep still and do not move the source and receiver positions. The standard deviation of time picks on horizons was as low as $22 \mu \mathrm{s}$, yielding an NRMS of $0.9 \%$ considering an effective peak frequency of $70 \mathrm{~Hz}$. Here, the system provided a sensitivity 1 order of magnitude higher than the best surface conventional acquisition. The time differences, measured between horizons above and below the reservoir, have been calibrated to the pressure changes in the reservoir measured in a well. The correlation looks excellent. The method is so sensitive that until sources and receivers were placed a few meters below the surface, the time-lapse changes were dominated by daily temperature effects.

Despite of all these early and more recent successes, we still have questions about the sensitivity of this method and how far we can go. Source stability, noise levels, consistency of the earth's properties along the path from source to target to receiver and consistency on the near surface and its influence on the ghost and other multiples were raised. Today, the main vulnerability of this measurement system seems to come from ghost and surface-multiple changes due to a changing overburden. 